\title{
BMJ Open Molidustat for the treatment of renal anaemia in patients with dialysis- dependent chronic kidney disease: design and rationale of three phase III studies
}

\author{
Tadao Akizawa, ${ }^{\oplus 1}$ Megumi Taguchi, ${ }^{2}$ Yoshimi Matsuda, ${ }^{3}$ Kazuma lekushi, ${ }^{2}$ \\ Takashi Yamada, ${ }^{4}$ Hiroyasu Yamamoto ${ }^{\circ}$
}

To cite: Akizawa T, Taguchi M, Matsuda Y, et al. Molidustat for the treatment of renal anaemia in patients with dialysis-dependent chronic kidney disease: design and rationale of three phase III studies. BMJ Open 2019;9:e026602. doi:10.1136/ bmjopen-2018-026602

- Prepublication history and additional material for this paper are available online. To view these files, please visit the journal online (http://dx.doi. org/10.1136/bmjopen-2018026602).

Received 11 September 2018 Revised 11 April 2019 Accepted 15 May 2019

\section{SLinked}

- http://dx.doi.org/10.1136/ bmjopen-2018-026704

Check for updates

(c) Author(s) (or their employer(s)) 2019. Re-use permitted under CC BY-NC. No commercial re-use. See rights and permissions. Published by BMJ.

For numbered affiliations see end of article.

Correspondence to Professor Tadao Akizawa; akizawa@med.showa-u.ac.jp

\section{ABSTRACT}

Introduction New medications for anaemia associated with chronic kidney disease (CKD) are desirable, owing to the limitations of erythropoiesis-stimulating agents (ESAs), the current standard of care. Molidustat is a novel hypoxiainducible factor prolyl-hydroxylase inhibitor that stimulates erythropoietin production, predominately in the kidney. We report methodological details of three phase III trials, named Molldustat once dailY improves renal Anaemia By Inducing erythropoietin (MIYABI), designed primarily to investigate the efficacy of molidustat therapy in adults with renal anaemia and dialysis-dependent CKD.

Methods and analysis MIYABI Haemodialysis-Correction (HD-C) is a single-arm trial (24-week treatment duration) in approximately 25 patients on haemodialysis, currently untreated with ESAs. MIYABI Peritoneal Dialysis (PD) is a single-arm trial (36 week treatment duration) in approximately 50 patients on peritoneal dialysis, treated or untreated with ESAs. MIYABI HaemodialysisMaintenance (HD-M) is a randomised, active-controlled, double-blinded, double-dummy trial (52-week treatment duration) comparing molidustat with darbepoetin alfa in approximately 225 patients on haemodialysis, treated with ESAs. Molidustat (starting dose $75 \mathrm{mg} /$ day) will be titrated 4-weekly to maintain haemoglobin in predetermined target ranges. The primary objective is to evaluate the efficacy of molidustat, using the following measures: the rate of rise in haemoglobin $(\mathrm{g} / \mathrm{L} /$ week) at the first dose change up to week 8 (MIYABI HD-C); responder rate (MIYABI HD-C and MIYABI PD); mean haemoglobin level during weeks 33-36 and non-inferiority to darbepoetin alfa shown by change in mean haemoglobin level from baseline (MIYABI HD-M). The secondary objectives are to assess safety, pharmacokinetics and pharmacodynamics. These trials will provide the first evaluations of molidustat therapy in patients receiving either peritoneal dialysis or currently untreated with ESAs on haemodialysis, and provide further evidence in patients treated with ESAs on haemodialysis. Ethics and dissemination The protocols were approved by ethics committees at all participating sites. The trials will be conducted in accordance with the Declaration of Helsinki and Good Clinical Practice. Results arising from these studies will be published in peer-reviewed journal(s).
Strengths and limitations of this study

- Due to recruitment feasibility limitations, MIYABI Haemodialysis-Correction and MIYABI Peritoneal Dialysis are single-arm, open-label studies.

- In MIYABI Haemodialysis-Maintenance (HD-M), a randomised, double-blind study, molidustat treatment will be directly compared with an ESA (darbepoetin alfa), the current standard of care for renal anaemia, and will build on the results of a previous open-label phase Ilb trial in patients on haemodialysis.

- The MIYABI HD-M trial will involve a larger patient population $(n=150)$ receiving a $75 \mathrm{mg}$ starting dose than in the phase $\mathrm{Ilb}$ trial.

- Treatment durations will be longer (eg, 52 weeks in MIYABI HD-M) than in the phase llb trial (16 weeks), although some molidustat-treated patients in the phase Illb trial $(n=57)$ continued treatment in an extension study for up to 36 months.

- These are the first studies to directly investigate the efficacy of molidustat therapy in patients on peritoneal dialysis and in patients currently untreated with ESAs on haemodialysis.

Trial registration numbers NCT03351166; Pre-results, NCT03418168; Pre-results, NCT03543657; Pre-results

\section{INTRODUCTION}

Anaemia is a common and serious complication of chronic kidney disease (CKD), ${ }^{1}$ which worsens as CKD progresses. ${ }^{24}$ The main cause of anaemia associated with CKD (also known as renal anaemia) is erythropoietin (EPO) deficiency. ${ }^{5}$

Treatment with erythropoiesis-stimulating agents (ESAs) is the current standard of care for renal anaemia. ${ }^{6}$ However, this approach has limitations. In 10\%-20\% of patients, irrespective of dialysis status, ESAs 
are ineffective at raising haemoglobin $(\mathrm{Hb})$ to prespecified levels. ${ }^{7-9}$ ESAs may also cause several adverse events (AEs), including development or worsening of hypertension, ${ }^{10-12}$ rare cases of antibody-mediated pure red cell aplasia, ${ }^{13}$ poor cardiovascular outcomes and death. ${ }^{14-16}$ In patients with cancer and anaemia, ESA use is associated with increased risk of thrombosis. ${ }^{17}$ These AEs may be related to injecting high doses of ESAs to achieve $\mathrm{Hb}$ targets $^{1517-19}$ and excessive increases in Hb levels. ${ }^{20}$

A new approach under investigation involves using small molecules to inhibit hypoxia-inducible factor prolyl-hydroxylases (HIF-PH), thereby inducing EPO production. In addition to addressing EPO deficiency, the main cause of renal anaemia, the therapeutic effect of HIF-PH inhibition may also be mediated by increasing the availability of iron for erythropoiesis, as indicated by reductions in hepcidin levels. ${ }^{21-26}$ These findings are particularly notable, given that functional iron deficiency may contribute to the inadequate responses that $10 \%-20 \%$ of patients experience during treatment with ESAs, even though these patients often receive intravenous iron supplementation. ${ }^{5-9}$ HIF-PH inhibition may theoretically also have a downside, because HIF transcriptionally upregulates a large number of genes; although EPO gene upregulation is helpful in treating anaemia associated with CKD, vascular endothelial growth factor (VEGF) upregulation could result in neoplasia and diabetic retinopathy. ${ }^{22}$ However, in clinical trials of HIF-PH inhibitors, no safety signals or changes in VEGF levels were reported. ${ }^{24-26}$

Molidustat, a novel, orally administered inhibitor of HIF-PH, induces circulating levels of EPO close to the normal physiological range, with high relative selectivity for the induction of EPO gene expression, predominately in the kidney. ${ }^{21}$ Results from preclinical ${ }^{21}$ and clinical studies $^{27}$ suggest that molidustat is a promising option for the treatment of EPO-sensitive anaemia in patients with CKD. In preclinical studies, molidustat restored renal EPO production with minor induction of hepatic EPO. Molidustat increased plasma EPO and EPO mRNA in the kidney and prevented decline in haematocrit and corrected decreases in $\mathrm{Hb}$ level. ${ }^{21}$ In a randomised, placebo-controlled, phase I study involving 59 healthy participants, single doses of molidustat $(5-50 \mathrm{mg})$ elicited a dose-dependent increase in EPO and were well tolerated. ${ }^{27}$ In three 16 -week, randomised, phase IIb, doseranging studies, comprising one study with patients on haemodialysis and two studies with patients not on dialysis, more than 400 patients with CKD were enrolled. These studies demonstrated that, during treatment with flexible-dose molidustat, $\mathrm{Hb}$ levels could be corrected relative to placebo or maintained at levels comparable to those in patients who continued treatment with ESAs, with manageable side effects. ${ }^{28}$ Comparable results and no significant safety concerns were observed in extension studies up to 36 months (unpublished data).

Based on the positive findings of the preclinical and phase IIb clinical studies, the MolIdustat once dailY improves renal Anaemia By Inducing EPO (MIYABI) programme of five phase III trials has been designed to investigate molidustat therapy further in patients with renal anaemia in Japan. Here, we report the methodological details of the three MIYABI trials in which the efficacy (up to 36 weeks), safety, pharmacokinetics and pharmacodynamics (up to 52 weeks) of molidustat therapy will be investigated in patients receiving dialysis. These three trials will provide the first evaluations of molidustat therapy in patients on peritoneal dialysis and in patients currently untreated with ESAs on haemodialysis, as well as extending the evidence in patients treated with ESAs on haemodialysis.

\section{METHODS AND PLANNED ANALYSES}

\section{Study designs, objectives and populations}

Each of the three phase III trials is a multicentre study conducted in adults aged 20 years or older with renal anaemia and dialysis-dependent CKD in Japan. In each trial, the primary objective is to evaluate the efficacy of molidustat in the respective patient populations and, in the MIYABI Haemodialysis Maintenance (HD-M) trial, to show non-inferiority to darbepoetin alfa. The secondary objectives of each trial are to evaluate the safety, tolerability, pharmacokinetics and pharmacodynamics of molidustat during the treatment periods. The three trials commenced in the first half of 2018 and have finished recruiting. The planned end dates for MIYABI Haemodialysis Correction (HD-C), MIYABI Peritoneal Dialysis (PD) and MIYABI HD-M are November 2018, August 2019 and December 2019, respectively. Patient eligibility was assessed during screening periods lasting up to 12 weeks in the MIYABI HD-C and MIYABI PD studies and up to 8 weeks in MIYABI HD-M. To be eligible, the mean of at least two $\mathrm{Hb}$ measurements (both taken before dialysis, at least 2 days apart, with the last measurement taken within 14 days before study drug assignment, and with a difference of less than $12 \mathrm{~g} / \mathrm{L}$ between the lowest and highest values) was required to lie within prespecified levels (table 1). The main inclusion criteria are summarised in table 1 . All inclusion and exclusion criteria are shown in online supplementary table 1 .

MIYABI HD-C is a single-arm study in patients on haemodialysis who are not currently treated with ESAs, with a 24 -week treatment duration (figure 1 and table 1 ). Japanese guidelines for the clinical evaluation of medications for renal anaemia recommend demonstrating efficacy in the correction and maintenance of renal anaemia in patients on dialysis, as well as in patients not on dialysis. ${ }^{29}$ However, the number of patients with renal anaemia on dialysis who do not receive ESAs is limited in Japan. A single-arm study design was chosen for MIYABI HD-C owing to the feasibility of patient recruitment. ${ }^{30}$

MIYABI PD is a single-arm study in patients on peritoneal dialysis who are treated or not treated with ESAs, with 
Table 1 Trial designs, patient populations and treatments

\section{MIYABI HD-C \\ (NCT03351166)}

\section{MIYABI PD}

(NCT03418168)

Single-arm, multicentre

\section{MIYABI HD-M \\ (NCT03543657)}

Randomised, active-controlled, doubleblinded, double-dummy, parallel-group, multicentre

\begin{tabular}{|c|c|}
\hline Trial design & Single-arm, multicentre \\
\hline Patient population & Men and women (aged $\geq 20$ year \\
\hline \multirow[t]{4}{*}{$\begin{array}{l}\text { Key inclusion } \\
\text { criteria }\end{array}$} & $\begin{array}{l}\text { Patients with ESKD on } \\
\text { haemodialysis at least weekly } \\
\text { for } \geq 2 \text { weeks }\end{array}$ \\
\hline & $\begin{array}{l}\text { Mean of the last two } \mathrm{Hb} \text { levels } \\
\text { between } \geq 80 \text { and }<100 \mathrm{~g} / \mathrm{L}\end{array}$ \\
\hline & $\begin{array}{l}\text { Not treated with ESAs during } \\
\text { the } 8 \text { weeks before study drug } \\
\text { assignment }\end{array}$ \\
\hline & $\begin{array}{l}\text { Not treated with HIF-PH } \\
\text { inhibitors during the } 8 \text { weeks } \\
\text { before study drug assignment }\end{array}$ \\
\hline Study treatments & $\begin{array}{l}\text { A starting dose of } 75 \mathrm{mg} \\
\text { molidustat } \mathrm{OD} \text {, titrated } \\
\text { based on } \mathrm{Hb} \text { response of the } \\
\text { previous dose. Planned doses } \\
\text { for titration are } 5,12.5,25,50 \text {, } \\
75,100,150 \text { and } 200 \mathrm{mg} \text { OD } \\
\mathrm{Hb} \text { target range } \geq 100 \text { to } \\
<120 \mathrm{~g} / \mathrm{L}\end{array}$ \\
\hline
\end{tabular}

Not treated with HIF-PH

inhibitors during the 8 weeks before study drug assignment

\section{A starting dose of $75 \mathrm{mg} \quad$ Two groups:}

molidustat OD, titrated

based on $\mathrm{Hb}$ response of the previous dose. Planned doses for titration are 5, 12.5, 25, 50, 75, 100, 150 and $200 \mathrm{mg}$ OD $\mathrm{Hb}$ target range $\geq 110$ to $<130 \mathrm{~g} / \mathrm{L}$ molidustat+darbepoetin alfa placebo, or molidustatplacebo+darbepoetin alfa A starting dose of $75 \mathrm{mg}$ molidustat or molidustat placebo OD, titrated based on $\mathrm{Hb}$ response of the previous dose. Planned doses for titration are 5 , $12.5,25,50,75,100,150$ and $200 \mathrm{mg}$ OD $\mathrm{Hb}$ target range $\geq 100$ to $<120 \mathrm{~g} / \mathrm{L}$ A starting dose of darbepoetin alfa or darbepoetin alfa placebo will be decided in accordance with the previous ESA dosages and schedule of once a week or every 2 weeks per Japanese label

\section{Treatment duration, 24}

*For patients washed out from ESAs, the mean $\mathrm{Hb}$ level before dialysis (at least two measurements taken $\geq 2$ days apart, assessed by the central laboratory) must have decreased by $\geq 05 \mathrm{~g} / \mathrm{L}$ after the last ESA administration, AND the interval from the last ESA administration to study drug assignment should be $>1$ week for epoetin alfa, $>2$ weeks for darbepoetin alfa or $>4$ weeks for epoetin beta pegol. ESA, erythropoiesis-stimulating agent; ESKD, end-stage kidney disease; Hb, haemoglobin; HD-C, Haemodialysis-Correction; HDM, Haemodialysis Maintenance; HIF-PH, hypoxia-inducible factor prolyl-hydroxylase; MIYABI, Molldustat once dailY improves renal Anaemia By Inducing erythropoietin; OD, once daily; PD, Peritoneal Dialysis.

a 36-week treatment duration (figure 1 and table 1). A single-arm study design was chosen for MIYABI PD owing to the limited number of peritoneal dialysis patients with renal anaemia in Japan.

MIYABI HD-M is a randomised, active-controlled, double-blinded, double-dummy, parallel-group study comparing molidustat with darbepoetin alfa in patients on haemodialysis who are treated with ESAs (figure 1 and table 1). The study has a treatment duration of 52 weeks. As most patients with renal anaemia on dialysis in Japan are treated with ESAs, it is feasible to recruit sufficient patients to perform a confirmatory, randomised, double-blinded trial of molidustat in this patient population. Therefore, in MIYABI HD-M, eligible patients will be randomised in a ratio of 2:1 to the molidustat group or darbepoetin alfa group. Allocation to treatment arms 
A

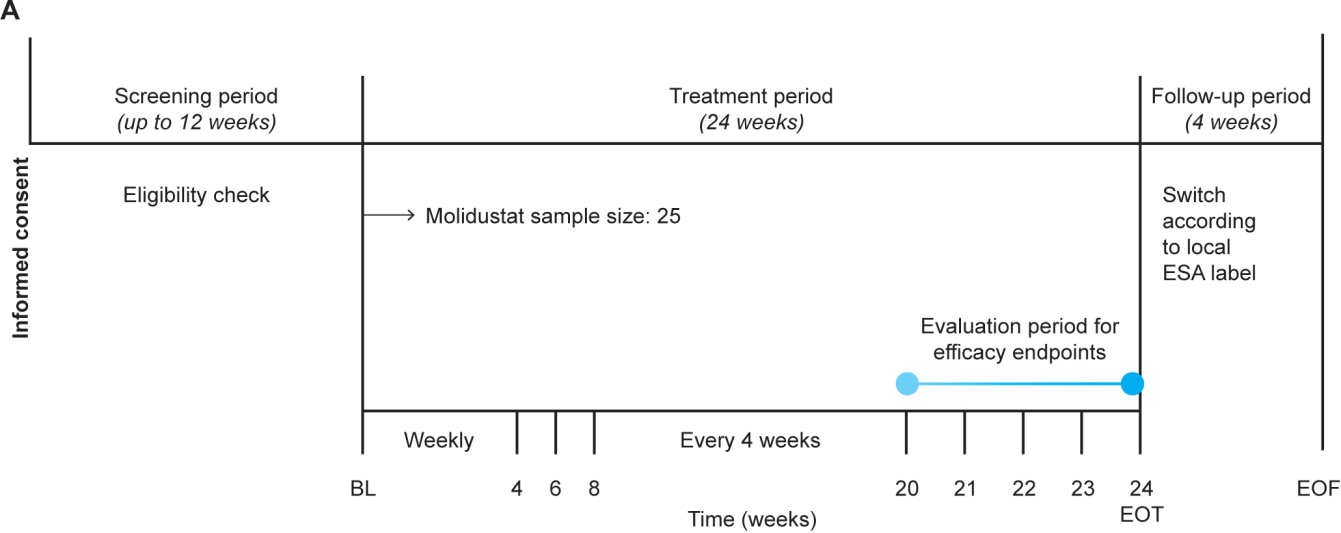

B

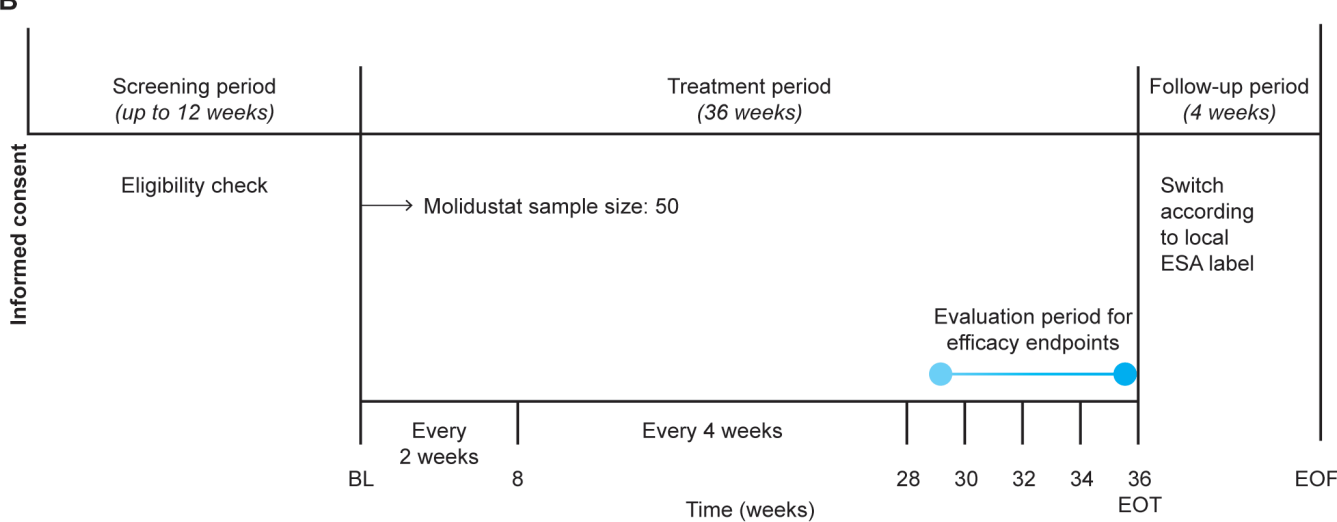

C

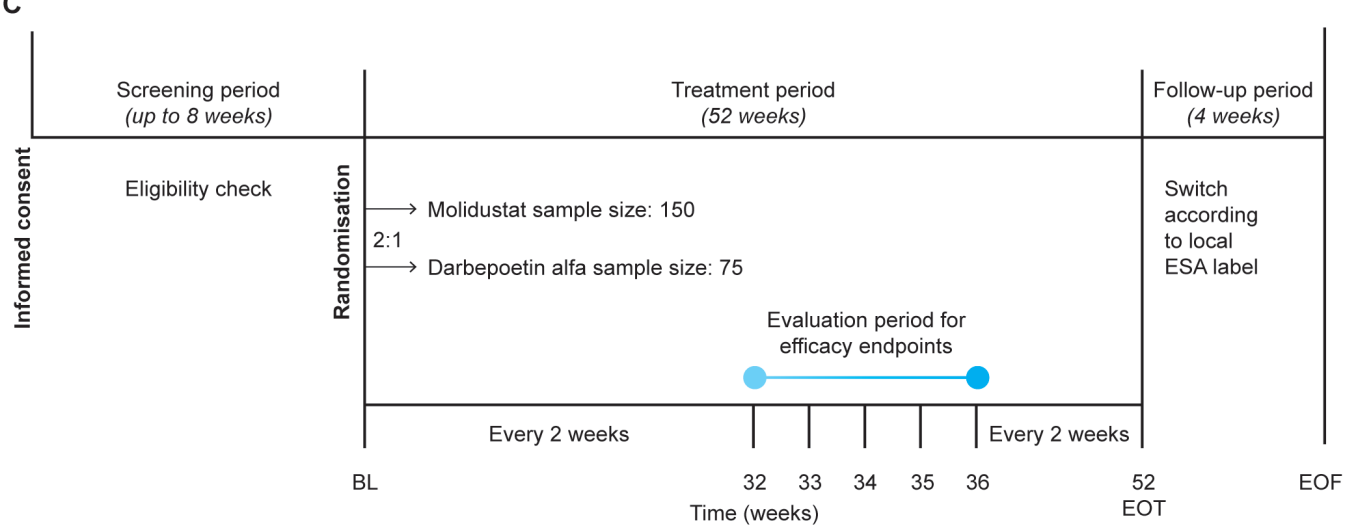

Figure 1 Trial designs for (A) MIYABI HD-C, (B) MIYABI PD and (C) MIYABI HD-M. MIYABI HD-C and MIYABI PD are singlearm, multicentre trials. MIYABI HD-M is a randomised, active-controlled, double-blinded, double-dummy, parallel-group, multicentre trial. Haemoglobin levels and safety will be assessed at each study visit, conducted at the time points shown. BL, baseline; EOF, end of follow-up; EOT, end of treatment; ESA, erythropoiesis-stimulating agents; HD-C, HaemodialysisCorrection; HD-M, Haemodialysis-Maintenance; MIYABI, Molldustat once dailY improves renal Anaemia By Inducing erythropoietin; PD, Peritoneal Dialysis.

will be achieved using an interactive voice/web response system (IxRS) at the first (baseline) visit. Randomisation will be stratified by previous ESA dose group (low or high) and by medical history of thromboembolic events (yes or no for myocardial infarction, pulmonary thromboembolism, stroke (excluding haemorrhagic stroke) or acute limb ischaemia). All investigators and patients in MIYABI HD-M will be blinded to treatment allocation. In cases of emergency, such as occurrence of a suspected, unexpected, serious $\mathrm{AE}$, when the investigator needs to know which drug has been allocated, unblinding will occur by entering the emergency key code for the relevant patient into the IxRS.

Each study is being overseen by a data monitoring committee consisting of independent clinical experts and an independent biostatistician supported by an independent statistical analysis centre, whose main responsibility is to recommend a change, interruption or termination of the study (or all phase three studies) based on safety findings. The data monitoring committee will be unblinded to treatment allocation in MIYABI HD-M. 
Treatments

Study treatments are summarised in table 1. In each study, a starting dose of $75 \mathrm{mg}$ molidustat once daily (OD) will be titrated every 4 weeks using the IxRS, based on the patient's $\mathrm{Hb}$ response to the previous dose. In each study, planned doses for the titration are 5, 12.5, 25, 50, 75, 100,150 and $200 \mathrm{mg}$ OD. The dose of molidustat will be adjusted to correct and maintain $\mathrm{Hb}$ levels in the target ranges of $\geq 100$ to $<120 \mathrm{~g} / \mathrm{L}$ in MIYABI HD-C and MIYABI HD-M and $\geq 110$ to $<130 \mathrm{~g} / \mathrm{L}$ in MIYABI PD, as per Japanese guideline recommendations. ${ }^{31}$

For patients untreated with ESAs (all patients in MIYABI HD-C and some patients in MIYABI PD, but no patients in MIYABI HD-M), a dose adaptation visit will occur at week 4 to avoid excessive elevation of $\mathrm{Hb}$ levels after the initiation of molidustat treatment. In MIYABI HD-C and MIYABI PD, dose titration at week 4 will be based on both the magnitude of the rise in $\mathrm{Hb}$ and the $\mathrm{Hb}$ level (see online supplementary table 2) and from week 8 according to the $\mathrm{Hb}$ level alone (see online supplementary table 3). For patients treated with ESAs (all patients in MIYABI HD-M and some patients in MIYABI PD, but no patients in MIYABI HD-C), the dose will be titrated from week 4 according to $\mathrm{Hb}$ level (see online supplementary table 3).

In MIYABI HD-M, patients will receive molidustat or molidustat placebo orally and darbepoetin alfa or darbepoetin alfa placebo intravenously. Patients in the molidustat group will receive molidustat plus darbepoetin alfa placebo, while patients in the darbepoetin alfa group will receive darbepoetin alfa plus molidustat placebo. The starting dose of darbepoetin alfa or darbepoetin alfa placebo will be selected for each patient based on their previous ESA dosage. Patients treated with darbepoetin alfa at screening will continue this treatment or start treatment with darbepoetin alfa placebo at the previous dose and interval (ie, weekly or biweekly). Patients treated with an epoetin therapy at screening will be treated with darbepoetin alfa or darbepoetin alfa placebo at a starting dose and interval determined by their epoetin dosage at screening. Then, depending on the $\mathrm{Hb}$ level (see online supplementary table 3), doses of darbepoetin alfa and darbepoetin alfa placebo will be titrated at biweekly intervals from week 2 , and doses of molidustat and molidustat placebo will be titrated from week 4 at 4-weekly intervals.

In all studies, in cases of excessive elevation of a patient's $\mathrm{Hb}$ level (rate of $\mathrm{Hb}$ rise $>10 \mathrm{~g} / \mathrm{L}$ per 2 weeks or $>20 \mathrm{~g} / \mathrm{L}$ per 4 weeks) during the treatment period, investigators may decrease the dose of molidustat (or darbepoetin alfa for MIYABI HD-M) at any time. If the administered dose is the minimum dose step, the dose may be suspended.

In each study, iron, vitamin B12 and folate supplementation is permitted if required and will be administered according to Japanese guideline recommendations. ${ }^{31}$ Iron supplementation will be administered to reach a target serum ferritin level of at least $100 \mathrm{ng} / \mathrm{mL}$ or transferrin saturation of at least $20 \%$.
For all studies, after the final administration of the study drug, further ESA treatment may be initiated at the discretion of the investigator. Details of the ESA treatment regimen will be recorded if treatment is initiated during the 4-week follow-up period.

\section{Variables}

All efficacy and safety variables, and associated definitions, are shown in table 2. The primary efficacy variables in MIYABI HD-C are the rate of rise in $\mathrm{Hb}$ level $(\mathrm{g} / \mathrm{L} /$ week) at the first dose change up to week 8 and responder rate. In MIYABI $\mathrm{PD}$, the primary efficacy variable will be the responder rate. In MIYABI HD-M, the primary efficacy variables will be mean $\mathrm{Hb}$ level during the evaluation period and its change from baseline. In all three studies, a responder is defined as a patient who meets all of the following criteria: (1) mean of the $\mathrm{Hb}$ levels during the evaluation period is in the target range; (2) $\geq 50 \%$ of the $\mathrm{Hb}$ levels during the evaluation period are in the target range; (3) no rescue treatment received up to the end of the evaluation period. Secondary variables for the three trials are shown in table 2. In each study, exploratory variables will include measures of iron metabolism, VEGF levels and assessment of health-related quality of life using the EuroQol 5-dimension 5-level questionnaire.

In each study, to investigate systemic exposure to molidustat and the relationship between molidustat exposure and response, sparse sampling from all patients will be conducted for pharmacokinetics and pharmacodynamics. If possible, molidustat exposure parameters (eg, $\left.\mathrm{C}_{\max }, \mathrm{AUC}\right)$ and the relationship between molidustat exposure and treatment effects will be evaluated using population approaches (eg, non-linear mixed effect modelling), including potential influence of relevant patient covariables.

\section{Quality assurance and data management}

For all studies, audits may be conducted by a member of the sponsor's quality assurance unit to assess the performance of the study at any of the study sites. In addition, sites may be inspected by regulatory health authority representatives, independent ethics committees and institutional review boards.

For all studies, data will be recorded by investigational site personnel onto the validated and password-protected electronic data capture system Rave (Medidata Solutions). All records identifying the patient will be kept confidential and will not be made available either to the public or the sponsor. All personal information made available for inspection will be handled in strictest confidence and in accordance with local data protection laws. Data will be pseudonymised for analysis. The sponsor will have access to the full trial data set.

The sponsor maintains clinical trial insurance coverage for each of the studies to provide compensation in the unlikely event that a patient is harmed from participation in any of these clinical trials. 
Table 2 Efficacy and safety variables

\begin{tabular}{llcl}
\hline & MIYABI HD-C & MIYABI PD & MIYABI HD-M \\
\hline $\begin{array}{l}\text { Primary efficacy variables } \\
\text { specific to each trial }\end{array}$ & $\begin{array}{l}\text { Rate of rise in Hb level (g/L/week) at } \\
\text { the first dose change up to week } 8^{*}\end{array}$ & $\begin{array}{l}\text { Responder rate during the } \\
\text { evaluation period (weeks } \\
30-36) \dagger\end{array}$ & $\begin{array}{l}\text { Mean Hb level during the } \\
\text { evaluation period (weeks } \\
33-36)\end{array}$ \\
& $\begin{array}{l}\text { Responder rate during the evaluation } \\
\text { period (weeks 21-24) } \dagger\end{array}$ & $\begin{array}{l}\text { Change in mean Hb level from } \\
\text { baseline during the evaluation } \\
\text { period }\end{array}$
\end{tabular}

$\begin{aligned} & \text { Secondary efficacy } \\ & \text { variables in all three trials }\end{aligned}$
Proportions of patients who meet the three response criteria during the evaluation period $\dagger$
Proportion of patients whose mean $\mathrm{Hb}$ level is in, above or below the target range during the evaluation period
Proportion of patients whose $\mathrm{Hb}$ level is in, above or below the target range, respectively, at each visit
change in $\mathrm{Hb}$ level/duration between two visits (weeks))
$\begin{aligned} & \text { Secondary efficacy } \\ & \text { variables specific to each } \\ & \text { trial }\end{aligned}$
$\begin{aligned} & \text { Rate of rise in } \mathrm{Hb}(\mathrm{g} / \mathrm{L} / \text { week) at the } \\ & \text { dose change up to week } 4\end{aligned}$

Other efficacy variables in MIYABI HD-C and MIYABI HD-M (secondary variables in MIYABI PD)

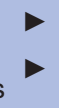

\section{Rate of rise in $\mathrm{Hb}$ level ( $\mathrm{g} / \mathrm{L} /$ week) during each visit interval}

- Percentage of days in the target $\mathrm{Hb}$ range during the evaluation period and treatment period, respectively (defined as the number of days in the target range/number of days during the period $\times 100$ [\%])

- Percentage of $\mathrm{Hb}$ levels in target range during the evaluation period and treatment period, respectively (defined as the number of measurements in the target range/number of measurements $\times 100[\%]$ )

- Proportion of patients who received at least one rescue treatment (RBC transfusion, ESA treatment)

Safety variables in all three trials

\section{Adjudicated AEsł}

- AEs including serious AEs

- Change in vital signs (pulse rate and blood pressure)

12-lead ECG parameters

- Observations of ophthalmological examination (fundus and anterior ocular segment examination and intraocular pressure measurement)

- Laboratory examinations (including haematology, coagulation, clinical chemistry, electrolyte, $\mathrm{HbA}_{1 \mathrm{c}}, \mathrm{PTH}$ and TSH levels)

Exploratory variables in all
three trials

${ }^{*}$ Rate of rise in $\mathrm{Hb}(\mathrm{g} / \mathrm{L} /$ week) at the first dose change up to week 8 is defined as the change in $\mathrm{Hb}$ level from baseline to the first dose change of study drug up to week 8 divided by the duration of the starting dose (in weeks). If no dose change is performed up to week 8 , then an $\mathrm{Hb}$ level at week 8 and the date of the week 8 visit will be used to calculate the change in $\mathrm{Hb}$ level and duration.

†A responder is defined as a patient who meets all of the following criteria: (1) mean of the Hb levels during the evaluation period is in the target range; (2) $\geq 50 \%$ of the $\mathrm{Hb}$ levels during the evaluation period are in the target range; (3) no rescue treatment up to the end of the evaluation period.

$\ddagger$ Adjudicated AEs include death, myocardial infarction, unstable angina pectoris, stroke or transient ischaemic attack, pulmonary thromboembolism or acute limb ischaemia.

AEs, adverse events; EQ-5D-5L, EuroQol 5-dimension 5-level questionnaire; ESA, erythropoiesis-stimulating agents; Hb, haemoglobin; $\mathrm{HbA1c}$, glycated haemoglobin; HD-C, Haemodialysis-Correction; HD-M, Haemodialysis Maintenance; MIYABI, Molldustat once dailY improves renal Anaemia By Inducing erythropoietin; PD, Peritoneal Dialysis PTH, parathyroid hormone; RBC, red blood cell; TSH, thyroidstimulating hormone.

\section{Statistical analysis}

All variables (including demographic and other baseline characteristics) will be analysed descriptively with appropriate statistical methods: categorical variables by frequency tables and continuous variables by summary statistics (mean, $\mathrm{SD}$, minimum, median and maximum).
Summary statistics will be presented for the original data as well as for the difference from baseline.

In each study, the primary analysis set for efficacy will be the full analysis set, which includes all patients assigned to treatment who have at least one baseline $\mathrm{Hb}$ level (ie, at least one $\mathrm{Hb}$ level before the first dose of the study drug). 
In MIYABI HD-C, the primary efficacy variables (rate of rise in $\mathrm{Hb}$ and responder rate) and their two-sided $95 \%$ CI will be estimated using one-sample $t$-statistics and the Clopper-Pearson method, respectively. In MIYABI PD, the primary efficacy variable (responder rate) and its two-sided 95\% CI will be estimated using the ClopperPearson method. In MIYABI HD-M, the primary efficacy variables (mean $\mathrm{Hb}$ level and change in mean $\mathrm{Hb}$ level) will be analysed by sequentially testing two hypotheses. In MIYABI HD-M, the primary objective will be achieved if the following two hypotheses are confirmed: (1) In the molidustat treatment group, the mean $\mathrm{Hb}$ level during the evaluation period (weeks 33-36) remains within the target range $(\geq 100$ to $<120 \mathrm{~g} / \mathrm{L})$. The mean $\mathrm{Hb}$ level in the molidustat treatment group will be calculated using the mean $\mathrm{Hb}$ level per patient. If the lower limit of the two-sided $95 \% \mathrm{CI}$ of the mean of the mean $\mathrm{Hb}$ level is greater than or equal to the lower limit of the target $\mathrm{Hb}$ level (ie, $\geq 100 \mathrm{~g} / \mathrm{L}$ ) and if the upper limit of the two-sided 95\% CI is less than the upper limit of the target $\mathrm{Hb}$ level (ie, $<120 \mathrm{~g} / \mathrm{L}$ ), it will be established that the mean $\mathrm{Hb}$ level is within the target range. Two-sided $95 \%$ CI will be estimated using one sample $t$-statistics. (2) Molidustat is not inferior to darbepoetin alfa. The non-inferiority of molidustat to darbepoetin alfa will be established if the lower limit of the two-sided 95\% CI for the difference (molidustat minus darbepoetin alfa) is above $-10 \mathrm{~g} / \mathrm{L}$ with non-inferiority margin of $10 \mathrm{~g} / \mathrm{L}$. This margin was chosen because a variation of approximately $10 \mathrm{~g} / \mathrm{L}$ is considered acceptable in Japanese clinical practice. ${ }^{31}$ In MIYABI HD-M, the difference in change between the treatment groups and its two-sided $95 \%$ CI will be estimated using an analysis of covariance model, including treatment group, previous ESA dose group (low/high) and previous thromboembolic events (yes/no) as fixed effects and baseline $\mathrm{Hb}$ level as a covariate.

Several descriptive and exploratory subgroup analyses are planned for all studies, including age, sex, baseline weight, prior thromboembolic event and main cause of CKD. Subgroup analysis will also be conducted for previous ESA dose group for MIYABI HD-M, for baseline $\mathrm{Hb}$ level and duration of dialysis for MIYABI HD-C and for pretreatment with ESAs at assignment and duration of dialysis for MIYABI PD.

\section{Determination of sample size}

In MIYABI HD-C and MIYABI PD, the respective sample sizes of approximately 25 and 50 patients are determined based on feasibility.

In MIYABI HD-M, the sample size of 150 patients in the molidustat group and 75 patients in the darbepoetin alfa group should result in sufficient data to assess the long-term safety of molidustat therapy, assuming a dropout rate of approximately $30 \%$. If 150 patients are randomised to the molidustat group, the power to establish that mean $\mathrm{Hb}$ levels are within target levels during the evaluation period is $\geq 98 \%$, assuming an SD of $13-15 \mathrm{~g} / \mathrm{L}$ from the previous phase IIb studies. This sample size has $>90 \%$ power to reject the null hypothesis that molidustat is inferior to darbepoetin alfa with a non-inferiority margin of $10 \mathrm{~g} / \mathrm{L}$ at a one-sided $2.5 \%$ significance level, assuming the expected difference between molidustat and darbepoetin alfa to be $0 \mathrm{~g} / \mathrm{L}$ and with a common SD of $13-15 \mathrm{~g} / \mathrm{L}$.

\section{Patient and public involvement}

Patients are not involved in the design and conduct of the studies.

\section{DISCUSSION}

Renal anaemia due to EPO deficiency is a common and serious complication of CKD. ${ }^{1}$ However, new approaches to the treatment of renal anaemia are needed, owing to safety issues and limitations with current treatments. Results from previous studies, including three phase IIb dose-ranging trials, suggest that molidustat is a promising option for the treatment of EPO-sensitive anaemia in patients with CKD.

At present, only one phase IIb trial assessing molidustat has been conducted in patients with renal anaemia who are on dialysis. It is anticipated that the three phase III trials described here will demonstrate the efficacy and safety of molidustat in patients with renal anaemia on dialysis, and that the trials will have the following strengths, relative to the one phase IIb trial conducted in patients on dialysis: (1) in MIYABI HD-M, molidustat treatment will be compared with an ESA (darbepoetin alfa), the current standard of care for renal anaemia, in a doubleblinded manner, whereas an open-label design was used in the phase IIb trial, in which molidustat treatment was compared with another ESA (epoetin); (2) a larger patient population in the MIYABI HD-M trial $(\mathrm{n}=150)$ receiving a starting dose of $75 \mathrm{mg}$ molidustat OD than in the phase IIb trial ( $\mathrm{n}=44$ of the 157 patients treated with molidustat received a $75 \mathrm{mg}$ starting dose); (3) the treatment periods will be longer (eg, 52 weeks in MIYABI HD-M) than in the phase IIb trial (16 weeks), although about one-third of the molidustat-treated patients in the phase IIb trial $(n=57)$ continued treatment in an extension study, with a duration of up to 36 months; (4) molidustat therapy will be investigated for the first time in patients who are not treated with ESAs on haemodialysis in the MIYABI HD-C trial, and MIYABI HD-M will provide further evaluations of molidustat in patients treated with ESAs, whereas the phase IIb trial only included patients who switched from epoetin; (5) the efficacy of molidustat therapy will be investigated for the first time in patients on peritoneal dialysis in MIYABI PD, whereas only patients undergoing haemodialysis were included in the phase IIb trial.

Approximately 600 patients are planned to be involved in the five studies in the phase III MIYABI programme; three studies in patients on dialysis and two studies in patients not on dialysis. While safety assessments will be conducted for all patients in the MIYABI programme, including assessments of vital signs and 12-lead ECG 
parameters, the sample size is insufficient to determine the risk of cardiovascular events. The MIYABI HD-C and MIYABI PD studies will also be limited to investigating molidustat therapy in the absence of a comparator and with small sample sizes (approximately 25 and 50, respectively), owing to the feasibility of patient recruitment (in Japan, limited numbers of patients with renal anaemia are on dialysis while not receiving ESAs or are on peritoneal dialysis), although molidustat will be compared with the current standard of care (darbepoetin alfa) as a maintenance treatment in a study powered to assess efficacy and safety in 150 patients on dialysis.

In the three MIYABI trials in patients on dialysis, the efficacy of molidustat will primarily be assessed by investigating $\mathrm{Hb}$ levels, including changes from baseline and maintenance of prespecified $\mathrm{Hb}$ targets. However, several exploratory variables will be also investigated. These include assessments of VEGF levels and ophthalmological examinations, conducted to evaluate the theoretical risk of VEGF-mediated diabetic retinopathy, ${ }^{22}$ and biomarkers of iron metabolism as, in addition to increasing EPO production predominately in the kidney, molidustat may increase the availability of iron for erythropoiesis. ${ }^{21-23}$

In summary, the three trials in patients on dialysis described here, together with two other trials in patients who are not receiving dialysis (the MIYABI ND-C and MIYABI ND-M randomised, open-label, active-controlled, parallel-group, multicentre trials), comprise the MIYABI phase III programme. The design and rationale of MIYABI ND-C and MIYABI ND-M are published in a companion article. ${ }^{32}$ This programme will investigate the efficacy and safety of molidustat in a broad clinical spectrum spanning approximately 600 patients with renal anaemia and CKD in Japan.

\section{Ethics and dissemination}

The studies are being conducted in accordance with the principles of the Declaration of Helsinki and the International Council for Harmonisation of Technical Requirements for Pharmaceuticals for Human Use Guidelines for Good Clinical Practice (GCP). Documented approval from appropriate independent ethics committees and institutional review boards has been obtained, according to GCP and local laws, regulations and organisations. The MIYABI HD-C study has been approved by the institutional review board of All Tohoku Clinical Trial Review and Audit Organisation (application number: 20171204) and another 20 sites. The MIYABI PD study has been approved by the institutional review board of Kyushu University Hospital (application number: 20180221) and another 26 sites. The MIYABI HD-M study has been approved by the institutional review board of Ibaraki Prefectural Central Hospital (application number: 20180524), Asahikawa-Kosei General Hospital (20180806) and another 51 sites.

Informed consent was obtained from patients by the site investigator or a designated person before entering the studies and may be withdrawn at any time. Proposed protocol amendments must be agreed by the sponsor and investigators and approved by independent ethics committees and institutional review boards. Protocol amendments must be signed by the principal investigator and have received all external approvals before coming into effect at the respective centre. If there is a change in the protocol that necessitates a change in consent, the investigator will inform patients of the changes in a timely manner and ask each patient to reconfirm their participation in the study by signing a revised consent form.

The studies have been registered on ClinicalTrials.gov (NCT03351166 [MIYABI HD-C], NCT03418168 [MIYABI PD], NCT03543657 [MIYABI HD-M]). Results will be disseminated through peer-reviewed publication(s) but there are no plans to publicly release the full protocol, participant-level data set or statistical code from any of the studies.

\section{Author affiliations}

${ }^{1}$ Division of Nephrology, Department of Medicine, Showa University School of Medicine, Tokyo, Japan

${ }^{2}$ MAF Pulmonology \& Cardiology, Medical Affairs, Bayer Yakuhin, Ltd, Osaka, Japan ${ }^{3}$ Statistics \& Data Insights, Data Sciences \& Analytics, Research \& Development, Bayer Yakuhin, Ltd, Osaka, Japan

${ }^{4}$ TA Thrombosis \& Nephrology, Clinical Development \& Operations, Research \& Development, Bayer Yakuhin, Ltd, Osaka, Japan

${ }^{5}$ Division of Nephrology and Hypertension, Department of Internal Medicine, The Jikei University School of Medicine, Tokyo, Japan

Acknowledgements Medical writing support was provided by Michael Riley, $\mathrm{PhD}$, of Oxford PharmaGenesis, UK, with funding from Bayer Yakuhin Ltd. Hitomi Mizutani, Eriko Ogura and Ken Miyazaki of Bayer Yakuhin Ltd reviewed the manuscript for statistical and/or scientific accuracy.

Contributors TA, HY and TY contributed to designing these studies. TY contributed to developing the original study protocols. MT contributed to drafting the article and revising it. TA, HY, MT and KI critical revised the article for important intellectual content. YM contributed to developing the statistical analysis plan and assisted in the preparation of the manuscript. All authors approved the final version of the manuscript and agree to be accountable for all aspects of the work, ensuring that questions related to the accuracy or integrity of any part of the work are appropriately resolved.

Funding These trials are funded by Bayer Yakuhin Ltd. The trials were designed and are being conducted by employees of Bayer Yakuhin Ltd, in consultation with healthcare professionals including TA and HY. Bayer Yakuhin Ltd is responsible for the design of these studies and the analysis and interpretation of data collected by investigators. Bayer Yakuhin Ltd and participating contract research organisations are responsible for management of data and writing the report. Bayer Yakuhin Ltd will make the final decision regarding submission of a manuscript for publication.

Competing interests MT, YM, Kl and TY are employees of Bayer Yakuhin Ltd. TA received consulting and lecture fees from Bayer Yakuhin Ltd during the conduct of the study. TA also received consulting, lecture or manuscript fees outside the submitted work from Astellas, GlaxoSmithKline, JT Pharmaceuticals, Kissei Pharmaceutical Co. Ltd, Kyowa Hakko Kirin, Nipro Corporation, Fuso Pharmaceutical Industries Ltd, and Ono Pharmaceutical Co. Ltd, and lecture fees from Bayer Yakuhin, Chugai Pharmaceutical Co. Ltd, Kyowa Hakko Kirin, and Torii Pharmaceutical Co. Ltd. HY received consulting fees from Bayer Yakuhin Ltd during the conduct of the study.

Patient consent for publication Not required.

Provenance and peer review Not commissioned; externally peer reviewed.

Open access This is an open access article distributed in accordance with the Creative Commons Attribution Non Commercial (CC BY-NC 4.0) license, which permits others to distribute, remix, adapt, build upon this work non-commercially, and license their derivative works on different terms, provided the original work is 
properly cited, appropriate credit is given, any changes made indicated, and the use is non-commercial. See: http://creativecommons.org/licenses/by-nc/4.0/.

\section{REFERENCES}

1. Culleton BF, Manns BJ, Zhang J, et al. Impact of anemia on hospitalization and mortality in older adults. Blood 2006;107:3841-6.

2. Hsu CY, McCulloch CE, Curhan GC. Epidemiology of anemia associated with chronic renal insufficiency among adults in the United States: results from the Third National Health and Nutrition Examination Survey. J Am Soc Nephrol 2002;13:504-10.

3. Astor BC, Muntner P, Levin A, et al. Association of kidney function with anemia: the Third National Health and Nutrition Examination Survey (1988-1994). Arch Intern Med 2002;162:1401-8.

4. El-Achkar TM, Ohmit SE, McCullough PA, et al. Higher prevalence of anemia with diabetes mellitus in moderate kidney insufficiency: The Kidney Early Evaluation Program. Kidney Int 2005;67:1483-8.

5. Babitt JL, Lin HY. Mechanisms of anemia in CKD. J Am Soc Nephrol 2012;23:1631-4.

6. Kidney Disease: Improving Global Outcomes (KDIGO) Anemia Work Group. Clinical practice guideline for anemia in chronic kidney disease. Kidney Int Supp/ 2012;2:279-335.

7. Luo J, Jensen DE, Maroni BJ, et al. Spectrum and Burden of Erythropoiesis-Stimulating Agent Hyporesponsiveness Among Contemporary Hemodialysis Patients. Am J Kidney Dis 2016;68:763-71.

8. Gilbertson DT, Peng Y, Arneson TJ, et al. Comparison of methodologies to define hemodialysis patients hyporesponsive to epoetin and impact on counts and characteristics. BMC Nephrol 2013;14:44.

9. Rossert J, Gassmann-Mayer C, Frei D, et al. Prevalence and predictors of epoetin hyporesponsiveness in chronic kidney disease patients. Nephrol Dial Transplant 2007;22:794.800.

10. Abraham PA, Macres MG. Blood pressure in hemodialysis patients during amelioration of anemia with erythropoietin. J Am Soc Nephrol 1991;2:927-36.

11. Maschio G. Erythropoietin and systemic hypertension. Nephrol Dial Transplant 1995;10 Suppl 2(Suppl 2):74-9.

12. Strippoli GF, Navaneethan SD, Craig JC. Haemoglobin and haematocrit targets for the anaemia of chronic kidney disease. Cochrane Database Syst Rev 2006:CD003967. Cd003967..

13. Locatelli F, Covic A, Eckardt KU, et al. Anaemia management in patients with chronic kidney disease: a position statement by the Anaemia Working Group of European Renal Best Practice (ERBP). Nephrol Dial Transplant 2009;24:348-54.

14. Pfeffer MA, Burdmann EA, Chen CY, et al. A trial of darbepoetin alfa in type 2 diabetes and chronic kidney disease. $N$ Engl J Med 2009;361:2019-32.

15. Phrommintikul A, Haas SJ, Elsik M, et al. Mortality and target haemoglobin concentrations in anaemic patients with chronic kidney disease treated with erythropoietin: a meta-analysis. Lancet 2007;369:381-8.

16. Akizawa $\mathrm{T}$, Okumura $\mathrm{H}$, Alexandre AF, et al. Burden of Anemia in Chronic Kidney Disease Patients in Japan: A Literature Review. Ther Apher Dial 2018;22:444-56.
17. Bohlius J, Wilson J, Seidenfeld J, et al. Recombinant human erythropoietins and cancer patients: updated meta-analysis of 57 studies including 9353 patients. J Natl Cancer Inst 2006;98:708-14.

18. Solomon SD, Uno H, Lewis EF, et al. Erythropoietic response and outcomes in kidney disease and type 2 diabetes. N Engl J Med 2010;363:1146-55.

19. Szczech LA, Barnhart HX, Inrig JK, et al. Secondary analysis of the $\mathrm{CHOIR}$ trial epoetin-alpha dose and achieved hemoglobin outcomes. Kidney Int 2008;74:791-8.

20. Unger EF, Thompson AM, Blank MJ, et al. Erythropoiesis-stimulating agents--time for a reevaluation. N Engl J Med 2010;362:189-92.

21. Flamme I, Oehme F, Ellinghaus $P$, et al. Mimicking hypoxia to treat anemia: HIF-stabilizer BAY 85-3934 (Molidustat) stimulates erythropoietin production without hypertensive effects. PLoS One 2014;9:e111838.

22. Gupta N, Wish JB. Hypoxia-Inducible Factor Prolyl Hydroxylase Inhibitors: A Potential New Treatment for Anemia in Patients With CKD. Am J Kidney Dis 2017;69:815-26.

23. Akizawa T, Macdougall IC, Berns JS, et al. SP334IRON Regulation by molidustat, bay 85-3934, a daily oral hypoxia-inducible factor prolyl hydroxylase inhibitor in patients with chronic kidney disease. Nephrology Dialysis Transplantation 2018;33:i457.

24. Besarab A, Provenzano R, Hertel J, et al. Randomized placebocontrolled dose-ranging and pharmacodynamics study of roxadustat (FG-4592) to treat anemia in nondialysis-dependent chronic kidney disease (NDD-CKD) patients. Nephrol Dial Transplant 2015;30:1665-73.

25. Martin ER, Smith MT, Maroni BJ, et al. Clinical Trial of Vadadustat in Patients with Anemia Secondary to Stage 3 or 4 Chronic Kidney Disease. Am J Nephrol 2017;45:380-8.

26. Pergola PE, Spinowitz BS, Hartman CS, et al. Vadadustat, a novel oral HIF stabilizer, provides effective anemia treatment in nondialysis-dependent chronic kidney disease. Kidney Int 2016;90:1115-22.

27. Böttcher M, Lentini S, Arens ER, et al. First-in-man-proof of concept study with molidustat: a novel selective oral HIF-prolyl hydroxylase inhibitor for the treatment of renal anaemia. $\mathrm{Br} J$ Clin Pharmacol 2018;84:1557-65.

28. Macdougall IC, Akizawa T, Berns JS, et al. Effects of Molidustat in the Treatment of Anemia in CKD. Clin J Am Soc Nephrol 2019;14:28-39.

29. Japanese Society of Nephrology. Guideline for clinical evaluation of therapeutic medicines on renal anemia. 2018 https://www.jsn.or.jp/ news/epo_guidline.pdf.

30. Japanese Society for Dialysis Therapy. An overview of regular dialysis treatment in Japan. 2012 http://docs.jsdt.or.jp/overview/ pdf2013/p051.pdf.

31. Tsubakihara Y, Nishi S, Akiba T, et al. 2008 Japanese Society for Dialysis Therapy: Guidelines for Renal Anemia in Chronic Kidney Disease. Therapeutic Apheresis and Dialysis 2010;14:240-75.

32. Yamamoto $H$, Taguchi M, Matsuda $Y$, et al. Molidustat for the treatment of renal anaemia in patients with non-dialysis-dependent chronic kidney disease: design and rationale of two phase 3 studies. BMJ Open 2019. 